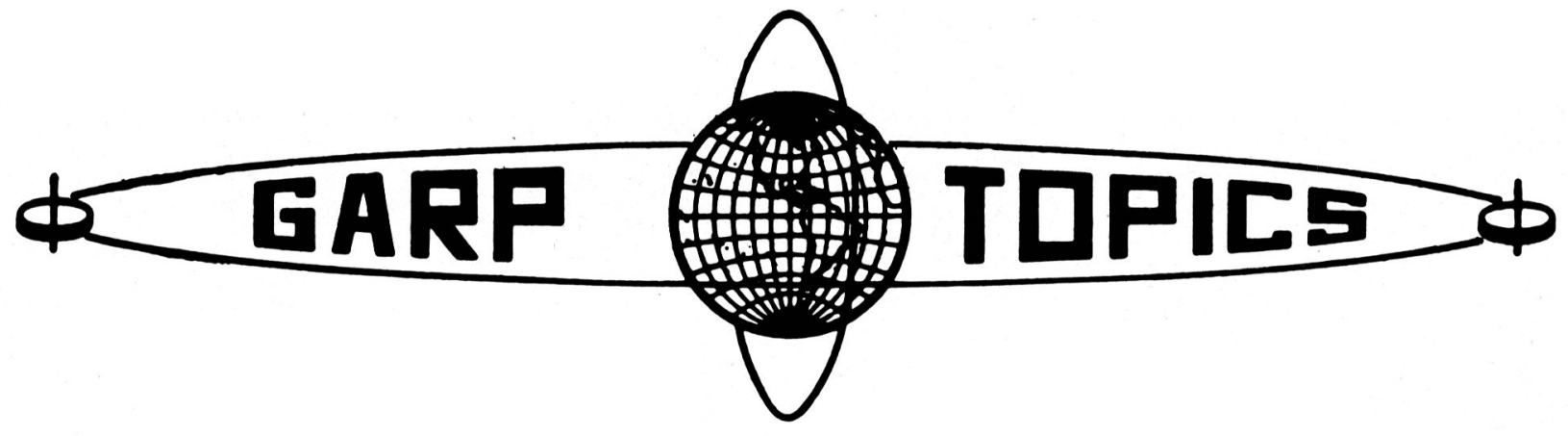

No. 75

June 1983

An occasional series reporting on U.S. and international GARP scientific, technical, and planning activities, developments, and programs, presented as a public service to the meteorological community by the American Meteorological Society through arrangements with the U.S. Committee on the Global Atmospheric Research Program of the National Academy of Sciences-National Research Council. Opinions expressed in "GARP Topics" do not necessarily reflect the point of view of the U.S. Committee.

\title{
The Time between a Field Experiment and its Published Results
}

\author{
Margaret A. LeMone \\ National Center for Atmospheric Research, ${ }^{1}$ Boulder, Colo. 80307
}

\begin{abstract}
A survey of the Journal of THE ATMOSPHERIC SCIENCES, the Journal of A pplied Meteorology, and the Monthly Weather REviEw shows that the number of publications per year resulting from GATE (GARP Atlantic Tropical Experiment) peaked in 1980 , six years after the experiment's field phase.
\end{abstract}

The purpose of this note is to show the surprisingly long time required for publication of the results of a major field experiment. The GATE (GARP Atlantic Tropical Experiment) meteorological experiment is chosen to demonstrate the time lag between the field experiment and the peak in the publications that result, since an acquaintance with both the scientists and subject matter involved facilitates scanning the journals for appropriate abstracts and titles. Papers from three journals are used: the JOURNAL OF THE ATMOSPHERIC SCIEnCES (JAS), the Journal of A PPlied METEOROLOGY

\footnotetext{
${ }^{1}$ The National Center for Atmospheric Research is sponsored by the National Science Foundation.

(c) 1983 American Meteorological Society
}

(JAM), and the Monthly Weather RevieW (MWR). There are several reasons for using just these three journals. First, the time lag, rather than the absolute number of papers, is of interest. Second, counting papers in conference proceedings often would result in counting the same paper more than once. And finally, although American papers do appear in them, journals published in other countries represent, to a large extent, groups of scientists subject to conditions different from their American colleagues-conditions that could result in different time lags.

To be counted, a paper had to rely primarily on GATE data or results. Papers that cite GATE data for a single point on a comprehensive graph, for example, were excluded. The selection procedure may be slightly nonuniform, since the journals have been perused sporadically over the last three years. However, the number of borderline papers is sufficiently small that the numbers in a given year are correct to within one or two papers. For 1981 at least, comments and responses are counted as one rather than two papers. Notes received the same weight as papers. The GATE bibliography that appeared in the last issue of the GATE Information Bulletin was not used, since it included much work by GATE investigators that was not related to GATE.

The number of papers by year for the three journals is presented in Fig. 1. Note the steady climb from 1974, the year 


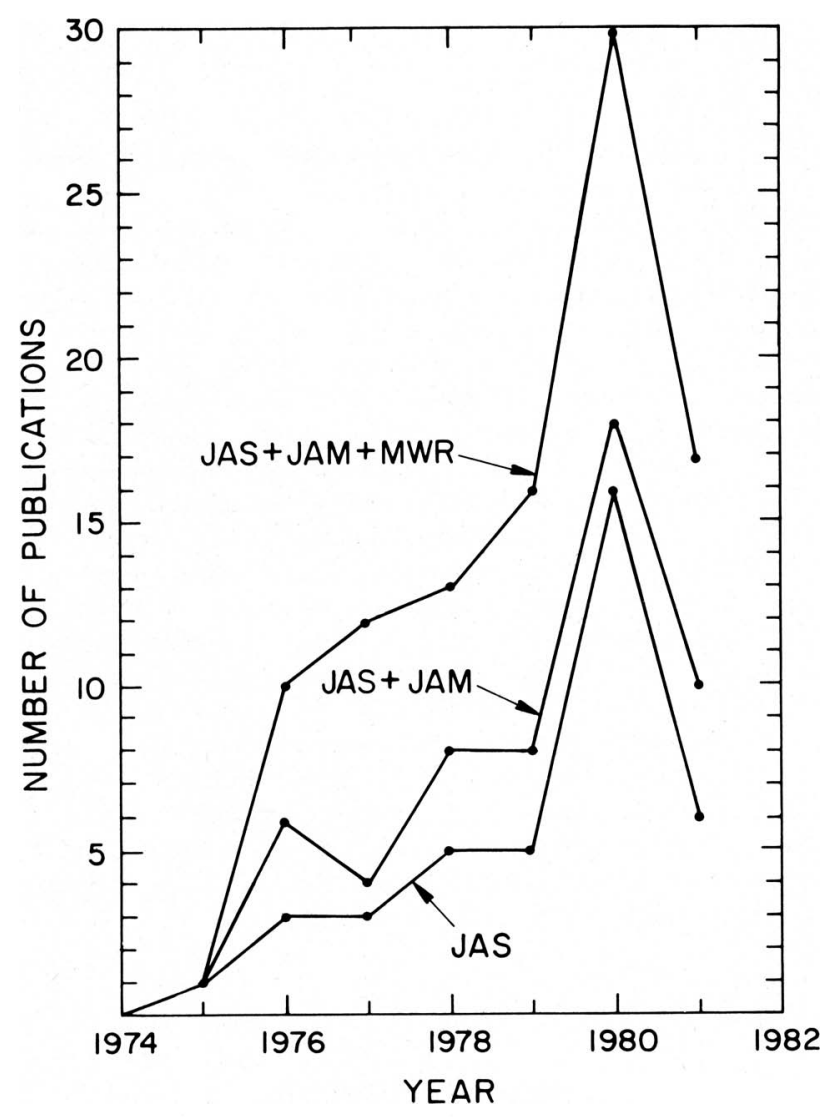

FIG. 1. Number of publications per year resulting from the GATE experiment, taken from the JOURNAL OF THE A TMOSPHERIC Sciences (JAS), the Journal of A pplied Meteorology (JAM), and the MONTHLy Weather REview (MWR). The lines on the diagram are cumulative as specified. of the experiment, to 1980. In 1981, the totals fell significantly, and the totals for 1982 through July (JAS, 3; JAM, 0; and MWR, 3) suggest a continued fall or a leveling off. Manuscripts circulating at present suggest the latter.

The types of papers varied with time. Earlier papers evaluated or used data from a single system, or used preliminary data, often from more conventional systems, such as radiosondes. The analysis typically was not sensitive to the problems in the data. Many could have been completed without the support GATE provided. But later papers depended on the comprehensive GATE data coverage, using data sets of increasing complexity (which also were available later), such as tethered balloons, radar, and aircraft. Use of these data without awareness of their unique problems, some of which surfaced during the GATE validation procedure, would have led to misleading results. Unconventional use of conventional data sets, such as carefully edited and filtered radiosonde data, for energy, momentum, and mass budgets also falls in this category. Some of the later papers use more than one data set and often involve more than one author. Review papers, the only contact with GATE for several scientists, are among the most recent.

The six-year lag between the completion of GATE and the peak in papers involving its results is surprising and significant. Similar studies should be done for other field experiments to see how size and other factors affect the time lag. Certainly, the results of very small experiments involving one or two investigators become published sooner after completion of the field phase. The time-lag figures then could be considered in the timing of new major field experiments and in the allocation of funds for analysis of the resulting data. Such figures also should make scientists involved in other field experiments feel a little better about the frustratingly long time required to publish their results. 